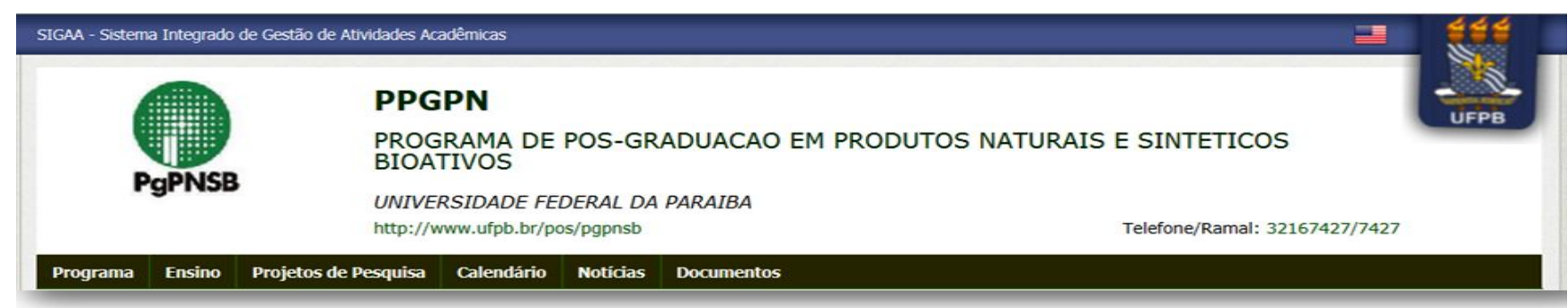

\title{
SciForum
}

MOL2NET

\section{Phytochemistry and anticancer potential of graviola (Annona muricata)}

\author{
Andressa Ferreira Viegas ${ }^{1 *}$, Thyago Fernandes dos Santos ${ }^{2}$, Liliane Campos Costa ${ }^{3}$ \\ 1 Centro Paraibano de Oncologia, Avenida Mato Grosso, n 183 - João Pessoa-PB; e-mail: \\ dressa.viegas@gmail.com \\ 2 Centro Paraibano de Oncologia, Avenida Mato Grosso, n 183 - João Pessoa-PB; e-mail: \\ thyago.fernandes@gmail.com \\ ${ }^{3}$ Centro Paraibano de Oncologia, Avenida Mato Grosso, n 183 - João Pessoa-PB. e-mail: \\ lilianecamposcosta@gmail.com
}

Received: / Accepted: / Published:

\begin{abstract}
Cancer is an invasive proliferative disease that is ranked among death leaders worldwide. There is a need in prospecting for novel therapeutic molecules, as well as the formulation of more effective chemotherapeutics. Since more than $40 \%$ of antineoplastics are derivative from natural products, the latter and their synthetic analogues have been widely used in this process, starting from simple to complex molecules, promoting specific target molecule targeting. The use of folk medicine helped in the discovery of a class with anticancer potential: acetogenin (AG). AG belong to the class of polyketides, present in the family Annonaceae. Thus, the objective of the present study was to carry out a review of prospective literature on the use of graviola (Annona muricata) with antitumor activity and future perspectives as chemotherapeutic. The review was conducted through the Medline/PubMed and Science Direct databases, selecting scientific articles on the subject, characterizing the literature review. The graviola has a quantity greater than 70 acetogenins (AG) that are distributed in the stem, leaves and seeds. AG has been shown to participate in the process of inducing death in tumor cells resistant to other chemotherapeutic agents. Many AG derivatives have shown toxicity against cancer cells, including multiple drug resistant (MDR) strains. Bullatacin, another type of AG, also showed marked antitumor activity against mammary cells with multiple drug resistance (MDR) phenotype. Another significant work demonstrated the participation of graviola AG in tumor cytotoxicity through inhibition of the mitochondrial complex, which is involved in oxidative phosphorylation and ATP synthesis. Furthermore, AG promotes apoptosis of breast tumor cells. Thus, the pharmaceutical technology in the formulation of $A G$ as a chemotherapeutic reveals a promising future in cancer treatment, especially the types that present the MDR phenotype, the biggest obstacle in cancer treatment.
\end{abstract}

Keywords: graviola; multidrug resistance; acetogenin; graviola and cancer. 


\section{Introduction}

Cancer is an invasive proliferative disease that is among the leaders of death in world, with cancer chemotherapy or also known as antineoplastic therapy being the primary form of treatment for malignant tumors. There is a need in the prospection of new therapeutic molecules, as well as the formulation of more effective chemotherapeutic drugs that overcome tumor resistance. According to Velingkar (2010), about 30 to $80 \%$ of cancers may become resistant to cytotoxic drugs, contributing to non-adherence to chemotherapeutic treatment. Tumor resistance in humans to various chemotherapeutic drugs is known as the main reason for the ineffectiveness of cancer therapy (Gottesman \& Pastan, 1993). Multiple drug resistance (MDR) is associated with high p-glycoprotein expression and remains the main challenge of effective cancer chemotherapeutic interventions (Ullah, 2008) because it limits the efficiency of several clinically important drugs allowing the some tumors the ability to survive high concentrations of cytotoxic drugs (Bellamy, 1996). Since more than $40 \%$ of antineoplastics are derived from natural products such as vinblastine extracted from

\section{Results and Discussion}

The graviola has a quantity greater than 70 acetogenins (AG) that are distributed in the stem, leaves and seeds. These are derivatives of long-chain (C32 or C34) fatty acids derived from the polyketide pathway, reviewed in (Liaw et al., 2016). Many of these derivatives are reported to be selectively toxic to cancer cells, including multidrug-resistant cancer cell lines. Recent studies have shown a marked antitumor effect belonging to the Annonaceae family. the Catharanthus roseus plant and paclitaxel extracted from the Taxus brevifolia plant, their synthetic analogs have been widely used in the molecular optimization process, from simple to complex molecules, promoting a specific target molecule targeting. The use of folk medicine through the preparation of infusions with the purpose of treating diseases aided in the discovery of a class with anticancer potential of the graviola (Annona muricata): acetogenina (AG). The AGs belong to the class of polyketides, present in the Annonaceae plant family, being the graviola integral to this family. The graviola is an evergreen plant of tropical and subtropical regions and is a rich source of natural products. The phytochemicals derived from graviola have various biological activities, including anticancer, antiarthritic, and antiparasitic activities. Thus, the objective of the present study was to perform a review of prospective literature on the use of graviola with antitumor activity and, especially, in resistant strains, inferring this plant as promising chemotherapeutic.

The results of such studies have demonstrated the effectiveness of the leaves in colon cancer strains. AG has been shown to participate in the process of inducing death in tumor cells resistant to other chemotherapeutic agents (Chang et al., 2001). Besides, many derivatives of AG have shown toxicity against cancer cells, including MDR strains (Moghadamtousi et al., 2015). Bullatacin, another type of AG, also showed marked antitumor activity 
against mammary cells with MDR phenotype. Another significant contribution was the work of McLaughlin (2008), demonstrating the participation of $\mathrm{AG}$ of graviola in tumor cytotoxicity through inhibition of the mitochondrial complex, which is involved in oxidative phosphorylation and ATP synthesis. According to the study by Kim (2018), the crude extract of graviola leaves induces apoptosis via the mitochondrial pathway and ceases cell proliferation, as well as reducing invasive capacity in breast tumor cells in a dose-dependent manner (Kim et al. 2018).

\section{Materials and Methods}

Scientific research on graviola and cancer was keywords were: graviola, graviola acetogenin, selected. The review was conducted through the graviola and cancer, resistance to multiple drugs.

Medline / PubMed and Science Direct databases, selecting scientific articles on the subject, characterizing the literature review. The

\section{Conclusions}

So far, the graviola is involved in antitumoral activity by inhibition of the EGFR and mithogens receptors, leading the dead of cancer (Rady et al. 2018). Thus, the pharmaceutical technology in the formulation of $\mathrm{AG}$ as a chemotherapeutic reveals a promising future in cancer treatment, especially the types that present the MDR phenotype, the biggest obstacle in cancer treatment.

\section{Conflicts of Interest}

The authors declare no conflict of interest in this paper.

\section{References and Notes}

Bellamy, W. T. P-glycoproteins and multidrug resistance. Annu Rev Pharmacol Toxicol:161-83, 1996.

Devita \& Chu. A history of cancer chemotherapy. Cancer Research. 68: 8643-8653, 2008.

Gottesman, M. M. ; Pastan, I. Biochemistry of multidrug resistance mediated by the multidrug transporter. Annu Rev Biochem:385-427, 1993.

Kim, Jee Young et al. 2018. "Annona Muricata Leaf Extract Triggered Intrinsic Apoptotic Pathway to Attenuate Cancerous Features of Triple Negative Breast Cancer MDA-MB-231 Cells.” 2018.

J. L. Mclaughlin, Journal Of Natural Products,2008, 71, 1311.

Liaw, Chih-chuang, Jing-ru Liou, Tung-ying Wu, and Fang-rong Chang. 2016. 101 Acetogenins from Annonaceae.

Mohd Fahad Ullah. Cancer Multidrug Resistance (MDR): A Major Impediment to Effective Chemotherapy. Asian Pacific J Cancer Prev, 9: 1-6, 2008.

Rady, Islam et al. 2018. "Review Article Anticancer Properties of Graviola ( Annona Muricata ): A Comprehensive Mechanistic Review.” 2018.

S. Zorofchian Moghadamtousi, H. Karimian, E. Rouhollahi, M. Paydar, M. Fadaeinasab, And H. Abdul Kadir, "Annona muricata leaves induce G1 cell cycle arrest and apoptosis 
through mitochondria-mediated pathway in human HCT-116 and HT-29 colon cancer cells," Journal of Ethnopharmacology, vol. 156, pp. 277-289, 2014.

S. Z. Moghadamtousi, m. Fadaeinasab, s. Nikzad, g. Mohan, h. M. Ali, and h. A. Kadir, "Annona muricata (Annonaceae): a review of its traditional uses, isolated acetogenins and biological activities", International Journal of Molecular Sciences, vol. 16, no. 7, pp. 15625-15658, 2015.

Velingkar V.S.; Dandekar V.D. Modulation of p-glycoprotein mediated multidrug resistance (MDR) in cancer using chemosensitizers. International Journal of Pharma Sciences and Research. 2: 104-111, 2010. 